\title{
THE COMPARATIVE BIOLOGY OF ANXIETY
}

\author{
By A. Comfort, M.A., M.B., D.C.H.
}

Anxiety is pre-eminently the psychical reaction which characterizes the human, as opposed to the subhuman mind, and the civilized as opposed to the savage man. The capacity for anxiety is closely parallel with intelligence-the Greek Prometheus ('forethought') who stole fire from heaven and was condemned to have his bowels gnawed by an eagle is a strikingly apt allegorical representation of fact. In the present social order, there is reason to believe that the physiological accompaniments of anxiety account for about 80 per cent. of the minor ailments, and at least 20 per cent. of the major ailments, attending hospital outpatient departments. It is in view of the growing importance of chronic anxiety as a medical problem that I am collecting here a few notes on its biological and neurological aspects, particularly its relation to attention behaviour in animals and man. If these are presented from the view point of zoology, not psychology, I can only justify myself by saying that the phylogeny of behaviour is possibly more evident today in society than ever before. The zoologist has a place in psychology which was perhaps more fully appreciated in the Igth century than it is today. The return to physical and physiological methods in psychiatry may perhaps tend to restore the balance.

\section{Anxiety and Fear in Animals}

Something like ' anxiety,' in the sense of a state of sensory and motor preparedness, is widespread among lower animals as the physiological state of attention. In all vertebrates whose behaviour is subcortically determined, it is possible to notice two or sometimes three states of sensorimotor tone; attention going on to alarm, a normal level and a quiescent state. The predominance of one or another level of attention varies from species to species, from one stage of growth to another in the same species, and according to cyclical (seasonal, day-night and habit) patterns. In birds, the three states are very obvious to the observer, and are characterized by difference in song. The quiescent state, or subattention, is characteristic of rest, feeding and mating. In the mating behaviour of most creatures, while sensory receptiveness is marked, it is concentrated on the business in hand. As the phylogenetic scale is ascended, the growth of the fore brain runs closely parallel with greater and greater variations in the level of attention which occur in the habits and behaviour of the animal, and an increasing power of selection of relevant stimuli from the continual sensory inflow of the environment. While the attention level of the lower animals (mollusca, annelids) is fairly constant, being varied only by definite defensive or hunting attention arising from direct stimuli, the attention level in mammals is capable of very wide variation indeed.

This attention pattern in any one animal depends on three main factors; its habits, its stage of development from birth to adulthood, and its metabolic cycle. The resting attention level is highest among animals which depend on speed (e.g. horses - the quagga and certain kinds of zebra have been known to die of acute hyperadrenalinism on being captured) and on hunting for survival. It is lowest in the forms which have the most effective physical defence (porcupines, skunks, and some arboreal forms -the latter develop a strikingly higher attention level when on the ground, and at a 
consequent disadvantage). Its ortogenic variation also depends on habit-it is higher in the leveret, which is born in a nest, than in the infant rabbit, which is born underground. It undergoes a superimposed metabolic cycle corresponding to day-night variation, seasonal activity, and senescence. It is modified in gregarious forms, where it ceases to be an ir.dividual activity; here the alarm pattern is an accentuation of individual alarm behaviour, the attention signal to other members of the herd. This alarm signal is not confined to herd-living animals, however, for it exists in solitary birds and even in those which are distributed on a territory system, and show no co-operative feeding or defence behaviour. The alarm system of ecological communities is as elaborate as any human air raid warning network, and there is an analogy between this mechanism and the extreme infectiousness of anxiety in human communities.

The pattern of the adult attention state depends largely on the length of parental protection. Attention behaviour is of a different kind in infancy, just as the first plumage and early protective colouring differ from the adult pattern. The infant deer has a spot and stripe pattern and a blotting reaction to alarm-the adult deer a different type of protective colouring and a two stage attention reaction, gaze followed by flight. The zoological aspect of the link between habit, degree of independence at birth and adult attention-alarm behaviour has been most fully worked out in birds, but the forms which this linkage takes are very similar in mammals.

In physical terms the attention reaction is the full adrenergic syndrome, arrection,' pressor response, inhibition of craniobulbar function including vagal tone, sensory hyperaesthesia, pupillary widening. Accordingly one might expect it to be least easily provoked when craniobulbar functions are predominant, during mating and feeding.

\section{Mating and Attention}

The attention pattern of most animals with a seasonal rut is, in fact, markedly altered during mating. Normal timidity may be largely lost, or it may be replaced by an aggressiveness which is in part a component of mating competition and in part, perhaps, a compensation for the change in normal alarm reactions. It is clear that if a high attention level depends upon, or is associated with, sympathotonia, either the attention pattern must alter during rut, or the normal habits must be varied to give some other security during mating. Most polyoestrous forms, and some others, specifically seek privacy to eat and copulate. This physical component of sexual modesty is not often stressed. It is also very striking that all-the-year-round breeding is characteristically, but not exclusively, found in animals which enjoy a definite ' home security,' rabbits, mice and cavies, but not hares. If, as I have suggested, the physiological patterns of attention and of mating are to some degree mutually exclusive, it is easy to see why the monoestrous tendency should go with a high attention level, in running and plain-dwelling forms, and the polyoestrous with a measure of physical security in hole dwellers. Zoologically speaking, there is evidence relating man to the second group. It is general experience that trains of instinctive behaviour, such as that of parenthood and mating, are aces capable of trumping the lower suits of self-preservation?

\section{Stimuli and Attention}

Attention has a large reflex component and the stimuli provoking attention have definite observable qualities in animals. The phenomena here rather suggest that while some variations in attention are innate, most are acquired. Most animals respond by an attention reaction to two main properties of any stimulus - discontinuity and unfamiliarity. All movement is capable of evoking attention in wild animals roughly in proportion to its suddenness, and the same applies to sound. Specific stimuli appear later by conditioning, but while a certain tendency to show alarm behaviour at any sudden stimulus is general in young animals, the subsequent conditioning is not individual, but largely caught from the behaviour of other members of the same species, or from the ecological community at large. This is noticeable in the change of attention level which is the essential feature of domestication. The sources of the con- 
ditioning which accompanies growth and experience are thus twofold-individual experience, coupled with a certain amount of instinctive behaviour. (how much is a matter of dispute) and group tradition based on imitation of parental, herd or ecological group attention and alarm. Young animals, and species such as the booby bird, have no group attention to the danger of man, but develop it rapidly as a result of individual experienceit is rather common for young animals and groups which are confronted with something wholly outside the group experience, such as the appearance of men, to react by curiosity rather than by alarm. The stimuli evoking curiosity are not well understood in animals, but they seem to be rather specific, and their use plays a part in the hunting behaviour of some predatory forms, e.g. snakes.

The feature of attention behaviour which seems to go most closely with the rise of cortical activity is the power of suspending external attention, or rather, of treating it selectively at a central level. The higher the degree of fore brain development, the greater the specificity of attention-producing stimuli, and the greater the preponderance, first of group, and then of individual conditioning. The work of Pavlov, who is the unchallenged pioneer of investigation of the attention behaviour of animals, and whose conclusions have not even now. been fully applied to ecology at large, provides the chief source of information about this growing specificity. The relevant points in Pavlovian psychology are the general specificity of sudden and unaccustomed sensory stimuli in evoking the attention state, the effect of repetition on this result, and the general inhibition (sleep) produced by monotonous reiteration of a stimulus which at first produced attention. Pavlov was also able to show that while pain is a very specific evocator of the attention-alarm response, it can be conditioned as a pleasure or 'vegetative' stimulus if it remains below a certain level of intensity-in other words, a pinprick or a blow which would produce an adrenergic effect in a normal animal can, by association with food, be made to produce a diametrically opposite effect; but that increase of intensity above a certain point reverses the process and restores the original response.
Now the attention state and the alarm state in animals, corresponding as they do to the 'red ' and ' purple' warnings with which we were familiar, are reactions which belong, in essence, to the rather clumsily named 'nocifensor' system, but it is associated not only with defence but with hunting behaviour, and accordingly we are probably more accurate in regarding its function as a preparation either for pleasure or for the avoidance of pain. In herbivora at least the nocifensor function predominates, and alarm states form part of a distinctive defence system which has medical significance-the most important effects of this system, from the point of view of anxiety and its symptoms in man, are tachycardia, intestinal inhibition, suppression of sexual behaviour, and arrection of hair. Moreover, while the attention and alarm states are closely comparable in most mammals, the special behaviour which is engrafted on them varies widely from form to form, and even, as we have seen, at different times in the same individual. The importance of this is that a stimulus which in the adult can produce general motor and sensory hyperactivity can, under slightly different circumstances or in the immature animal, produce a reflex central inhibition and immobility, which in some animals is so strong as to prevent any escape if they are once detected. Tonic and atonic responses to alarm bear rather a close resemblance to the tonic and atonic responses which characterize human anxiety in different patients.

\section{Relation of Attention States to Anxiety}

Although the physical manifestations of alarm in animals are well known to be closely parallel to those of psychogenic anxiety in man it is as rash to press this analogy from zoology to psychology as it is to apply any animal data to human beings. I have kept the terms attention and alarm for the psychophysical processes in animals because they beg no questions in this connection. Fear, which is frequently used as synonymous with alarm, carries the definite connotation of alarm plus unpleasant effect, and ought to be kept for the response of those animals in which effect sensu stricto can be said to exist, since zoologists 
and even psychologists are not always free of a certain anthropomorphic attitude to animal behaviour. Unpleasant effect may in itself be a physiological response to the obstruction of a pattern of instinctive behaviour, but it is doubtful at what evolutionary level true effect can be said to appear, although the subjective element in anxiety which distinguishes it from alarm is almost certainly not cortical. ${ }^{1}$ Where anxiety differs from fear on the one hand and alarm on the other is that it is a chronic prolongation of a process which is normally and physiologically acute. In watching the behaviour of birds and mammals one cannot resist the conclusion that each species and each individual has a definite 'setting' of the normal attention level, which is suited to its characteristics and habits. This is reflected in the relative ease with which some forms and some individuals are domesticated, while others of comparable learning power are not. The higher the initial non-specific setting, the harder it is to remove the effectiveness of stimuli by conditioning. Anxiety in man, as it presents to the psychiatrist, would probably appear to the general zoologist as the chronic heightening of this nonspecific reactivity which obstructs the power of learning and acts as a barrier to intelligence. Now we know from Pavlov that a comparable state can be produced artificially in dogs by contradictory conditioning, and that it abolishes not only specificity of alarm reactions but conditioning in general, but there is no evidence that this ever occurs in the wild state. The unique feature of human anxiety is that the stepping-up of the alarm level and the loss of specificity to stimuli is the result of an endogenous process-in other words, events occurring in the 'higher' areas can fire off or simulate the alarm response and the 'danger' effect which goes with it from above instead of from below, i.e. peripherally. This is a conclusion on which prefrontal lobotomy may shed a good deal of light. Alarm in animals is without significant exception reflex from the periphery, even when it is mediated through higher levels or 'selected' by them. The breakdown of this selectivity is conspicuous in very acute and severe anxiety attacks in mansome of the 'bomb happy' pass from an initial phase, in which every stimulus, irres- pective of origin, evokes a typical alarm response, to a more chronic phase in which the general level of attention alarm is raised and even normal proprioceptive sensations are interpreted as pain. The condition of 'pains all over' is not to be too cynically interpreted when it is associated, as it almost always is, with anxiety.

The phylogeny of effect should perhaps lead us to regard it as wholly a function of these instinctive patterns - unpleasant when the instinctive ' relay' is obstructed, pleasant when it goes to completion. This is the conclusion of much modern theory which has long abandoned the Spencerian approach to psychology via zoology, but to the zoologist the growth of the pattern, up to the point at which the endogenous sources of effective colour take over, is probably more obvious than it is to the clinician.

The zoologist approaches human psychology with these conclusions-man is ecologically gregarious, breeds all the year round, is dependent on parental care for longer than any other animal, has an indefinite alarng pattern which varies considerably in in dividuals, but which is closer to the herbivore than the carnivore pattern in that the alarm state is most usually, in civilized men at least, accompanied by an effect likely to lead to flight when unopposed, aggressive responses are secondarily developed when flight is obstructed; shows ontogenic development from a stage at which the fear reaction is accompanied by loud cries to attract the attention of parents to one in which flight predominates over fear, with some variation in the alarm response in pregnancy and parenthood but little in mating; and is capable of producing or activating the alarm mechanism or keeping it chronically stimulated by purely endogenous processes. This conception fits in with the known psychiatric facts and with o Pavlov's experiments-the alarm to higher process system will work in either direction, upwards, as it does in animals, so that an environment full of threatening stimuli gradually raises the level of alarm response, and downwards when a collision between two sets of conditioned stimuli, or a psychical ' conflict,' the nature of which it is not yet possible to 
express in terms of neurology, activates the alarm response from above, and with it, most commonly, the unpleasant effect which is functionally akin to it. Some part at any rate of the physical in psychical conflicts is wrapped up with this association between the unpleasant effect, the physiological pointer to that which is better avoided, and alarm ; we know that in animals any obstruction of the specific escape behaviour greatly exaggerates the alarm response, and this is equally true in man, when the obstruction is derived from the conscious level or from the standards set by superconsciousness or group convention. The zoologist would expect, on a basis of observation, many of the conclusions of psychiatry -that obstruction of any instinctive or acquired behaviour pattern, especially when it is associated with the physical and effective mechanism of danger and escape, would lead to a heightening and a decreased specificity in the alarm state. The reaction of the hare caught in a net, which responds either by heightened alarm response and ineffectual running movements, or by an immobility thich is probably a reversion to the infantile blotting pattern, is biologically comparable with the response of the man whom the net of civilized inhibition prevents from murduring his unwanted relatives, deserting his wife, or escaping from the battle front of a conflict in which he feels himself unconcerned. The running response and the pseudocatatonia were familiar enough in wartime--a more chronic heightening of the alarm level is perhaps the chief specific disease of urban civilization. At the same level, zoology has a close parallel for the communicability of alarm, especially from parent to child and among the members of a crowd. It is conspicuous in a series of 'welfare' cases how completely the response or non-response of the child to bombing depended on the amount of anxiety displayed by the parents, and how much less effective bombing, with its attendant curiosity reaction, proved than parental neurosis in inducing insecurity in the child. The behaviour of crowds, as exemplified at Bolton and in the history of panic under very various circumstances, is almost always better interpreted at the zoological level than the psychological.
Specific Physical Results of the 'Alarm State

A society in which alarm is endemic has its own diseases, and we can relate them to the nocifensor mechanisms I have described. Intestinal inhibition has its parallel in gastrointestinal neurosis, and probably in most cases of peptic ulcer (Winser, 1941). Pressor stimulation in certain cases corresponds to endemic hypertension. Sexual inhibition corresponds to female frigidity and male premature orgasm, and by a reverse mechanism inadequate detumescence is an observable cause of sympathotonia and anxiety. Much as one must suspect some published interpretations of this, there is a relationship, though not necessarily a causal one, between sexual unfulfilment and increased psychomotor tone. Perhaps the most interesting relationship is between arrection and skin disease; the parallel is tempting, in view of the close psychophysical relationship and the universality of phenobarbitone in dermatology. One would expect a society in which anxiety is endemic to be frigid, dyspeptic and constipated-a glance at the outpatient book or the page of quack medical advertisements confirms the conclusion.

\section{Conclusions}

To understand the external stimuli of this endemic anxiety one must go outside psychiatry to the work of sociologists like Lewis Mumford. The lack of rapport between psychiatric knowledge and the general study of cultures is rather striking, and even those who, like Halliday, ${ }^{2}$ have attempted to discover an epidemiological basis for anxiety states, show a certain unwillingness to plunge into a subject which is bound, at some point, to involve them in politics. Halliday, it is true makes it quite clear than de-industrialization is not a practical prophylaxis, though he does not answer the view that societies in which anxiety is endemic are showing every sign of being about to de-industrialize themselves by a process of increasing militarism and increasing vulnerability. The bases of megalopolitan anxiety were fully expounded by Mumford before conscription and atomic bombs were added to the load of specific 
stimuli. There is zoological and neurological reason to suppose that the purely atavistic alarm mechanisms are always most active in societies which have abolished individual purpose and responsibility and substituted herd obedience and a co-existent loneliness. The general biologist can help best by pointing to the very full discussion of the zoological traditions of social behaviour in the work of Kropotkin, a writer to whom Mumford owes much; and by urging on the psychiatrists a cautious attitude toward the creation of morale in a social order whose effects are very far from normal or desirable. There has been a deplorable tendency in wartime to use " adaptation ' in a tradition which belongs to Procrustes rather than Hippocrates, and the psychiatrist who considers Kropotkin convincing will find himself committed to a view of normality which includes the therapeutic use of individual disobedience. The desirability of being 'adapted' to a particular environment depends wholly on the desirability or other- wise of the environment itself. Such social $\stackrel{0}{0}$ environments as war or Naziism should themselves be 'adapted,' out of existence if $\frac{0}{3}$ possible, and it is poor medicine to attempt to $\stackrel{\mathbb{\alpha}}{\stackrel{\Phi}{2}}$ make normal persons acquiescent in them. We $C$. are largely unaware, under the conception of $\overrightarrow{\vec{F}}$ public order, how large a part of the cohesive force in our own society is based on the stimulation of fear-apart from the dangers $\overline{\bar{p}}$ and noise inherent in neotechnic life, and its $ه$ fantastic insecurity, there is hardly a single political or commercial agency which does not appeal to the public directly by way of the? creation of an alarm response-this is true $\vec{\omega}$ from 'blood, tears and sweat' down to the $\frac{\rho}{\partial}$ puffing of deodorants. 'On ne s'associe pas pour mourir,' said Espinas, and a psychiatry which intends to abolish the morbid expressions of anxiety will require to reassess its of relationship to any social order which, by a i reliance upon fear as a technique of persuasion, $\overrightarrow{0}$

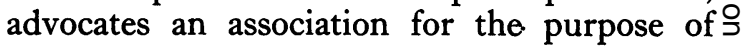
death rather than mutually co-operative life. 